\title{
Imaging molecular potentials using ultrahigh-resolution resonant photoemission
}

\author{
Catalin Miron ${ }^{1 \star}$, Christophe Nicolas ${ }^{1}$, Oksana Travnikova ${ }^{1}$, Paul Morin ${ }^{1}$, Yuping Sun ${ }^{2}$, \\ Faris Gel'mukhanov ${ }^{2}$, Nobuhiro Kosugi ${ }^{3}$ and Victor Kimberg ${ }^{1}$
}

\begin{abstract}
Electron-density distributions and potential-energy surfaces are important for predicting the physical properties and chemical reactivity of molecular systems. Whereas angle-resolved photoelectron spectroscopy enables the reconstruction of molecular-orbital densities of condensed species ${ }^{1}$, absorption or traditional photoelectron spectroscopy are widely employed to study molecular potentials of isolated species. However, the information they provide is often limited because not all vibrational substates are excited near the vertical electronic transitions from the ground state. Moreover, many electronic states cannot be observed owing to selection rules or low transition probabilities. In many other cases, the extraction of the potentials is impossible owing to the high densities of overlapping electronic states. Here we use resonant photoemission spectroscopy, where the absence of strict dipole selection rules in Auger decay enables access to a larger number of final states as compared with radiative decay. Furthermore, by populating highly excited vibrational substates in the intermediate coreexcited state, it is possible to 'pull out' molecular states that were hidden by overlapping spectral regions before.
\end{abstract}

Rydberg-Klein-Rees ${ }^{2}$ is one of the most popular methods to reconstruct molecular potentials for stable states of diatomic molecules from rovibrational spectroscopy data without requiring any assumption about the shape of the potential. It is in a way a complementary method to the direct imaging of molecular orbitals to study molecular structures ${ }^{3}$. Alternative approaches have been proposed on the basis of a fitting procedure of analytical potential functions to experimental data ${ }^{2,4}$. Here we show how powerful the use of core excitation can be for extracting molecular-potential curves of ionic states exclusively on the basis of ultrahigh-resolution resonant photoemission (RPE) data as obtained for $\mathrm{N}_{2}$. This has been achieved when controlling the extension of vibrational wavefunctions by fine tuning the X-ray energy. Indeed, tuning of the X-ray energy enables the spatial distribution of the vibrational wavefunctions in the intermediate state to be selected, and thus the extension of the probed region of the final-state potential to be controlled. Here we report the observation of the $1^{2} \Phi_{g}$ and $1^{2} \Delta_{u}$ states of $\mathrm{N}_{2}^{+}$and the accurate reconstruction of the $1^{2} \Pi_{g}$ and $1^{2} \Phi_{g}$ molecular potentials, demonstrating that state-of-the-art RPE spectroscopy can be successfully used to solve the problem of potential reconstruction for excited molecular states directly from experimental data, and to identify such new states, invisible in conventional photoemission spectroscopy.

Unlike direct photoemission, where the absorption of an X-ray photon of energy $\hbar \omega$ (where $\omega$ is the X-ray frequency) leads to the emission of a photoelectron, in the RPE process the resonant absorption of an X-ray photon populates final ionic states of the molecule by ejecting resonant Auger electrons with kinetic energies $E_{\mathrm{k}}$. Our measurements were made in the sublifetime regime (X-ray spectral bandwidth and spectrometer resolution considerably lower than the natural lifetime broadening $115 \pm 4 \mathrm{meV}$ (ref. 5), enabling unprecedented total linewidth of less than $52 \mathrm{meV}$; Methods), following selective vibrational excitation within the $\mathrm{N} 1 s \rightarrow \pi^{*}$ transition in gas-phase nitrogen molecules. The data were collected at the soft-X-ray PLEIADES beamline dedicated to the investigation of isolated species, at the synchrotron SOLEIL, France. This state-of-the-art spectroscopy facility, briefly described in refs 6,7, benefits from the improved spectral brightness of SOLEIL combined with innovative X-ray optics and instrumentation (Supplementary Information), and provides a unique opportunity to make highly demanding measurements. Indeed, in the present case its exceptional performance has enabled us to overcome the low number of ejected electrons due to (1) the normally low number of $\mathrm{X}$-ray photons in the very narrow bandwidth and (2) the significant drop, of more than one order of magnitude, of the photoexcitation cross-section in the region far above the vertical transition from the ground-state geometry. However, reaching the highly excited and almost invisible vibrational level $v=6$ in the $1 s^{-1} \pi^{*}$ state has been crucial to the full exploration of the molecular potentials. On the basis of these conditions we have been able to solve the inverse problem of molecular potential reconstruction using ultrahigh-resolution RPE spectra obtained over a broad range of $\mathrm{X}$-ray energies, enabling the size of the vibrational wavefunctions in the core-excited state to be accurately targeted. This Letter explains how this goal was achieved.

Figure 1 shows the $a b$ initio calculated potentials (Methods) of the molecular ion $\mathrm{N}_{2}{ }^{+}$(centre panel) together with the experimental photoemission (left-hand panel) and RPE (righthand panel) spectra plotted on a binding-energy scale $E_{\mathrm{b}}=\hbar \omega-E_{\mathrm{k}}$. The RPE spectrum shown was obtained when the X-ray energy was tuned to the $v=0$ vibrational substate of the $1 s^{-1} \pi^{*}$ state. Calculations (Methods) indicate a remarkably high density of final states in the region $E_{\mathrm{b}}=24-30 \mathrm{eV}$. Indeed, ten final electronic states build up the RPE spectrum (see Fig. 1 for the assignment of the individual bands). Partial contributions to the RPE spectrum drawn as coloured areas in Fig. 1 show the strong overlap of these ten bands associated with Auger decays, to either bound or dissociative (high$E_{\mathrm{b}}$ region) final states. Consequently, the first impression might be that RPE does not enable the accurate characterization of molecular potentials to be made. Nevertheless, looking at the weak spectral feature below $E_{\mathrm{b}} \approx 23.5 \mathrm{eV}$ in the bottom spectrum $(v=0)$ of Fig. 2 and its evolution when RPE spectra are recorded for a selection of X-ray energies successively tuned in resonance with the next six vibrational substates $v=1-6$ of the $1 s^{-1} \pi^{*}$ core-excited state

${ }^{1}$ Synchrotron SOLEIL, I'Orme des Merisiers, Saint-Aubin, BP 48, 91192 Gif-sur-Yvette Cedex, France, ${ }^{2}$ Theoretical Chemistry, Royal Institute of Technology, S-106 91 Stockholm, Sweden, ${ }^{3}$ Institute for Molecular Science, Myodaiji, 444-8585 Okazaki, Japan. *e-mail: Catalin.Miron@synchrotron-soleil.fr. 


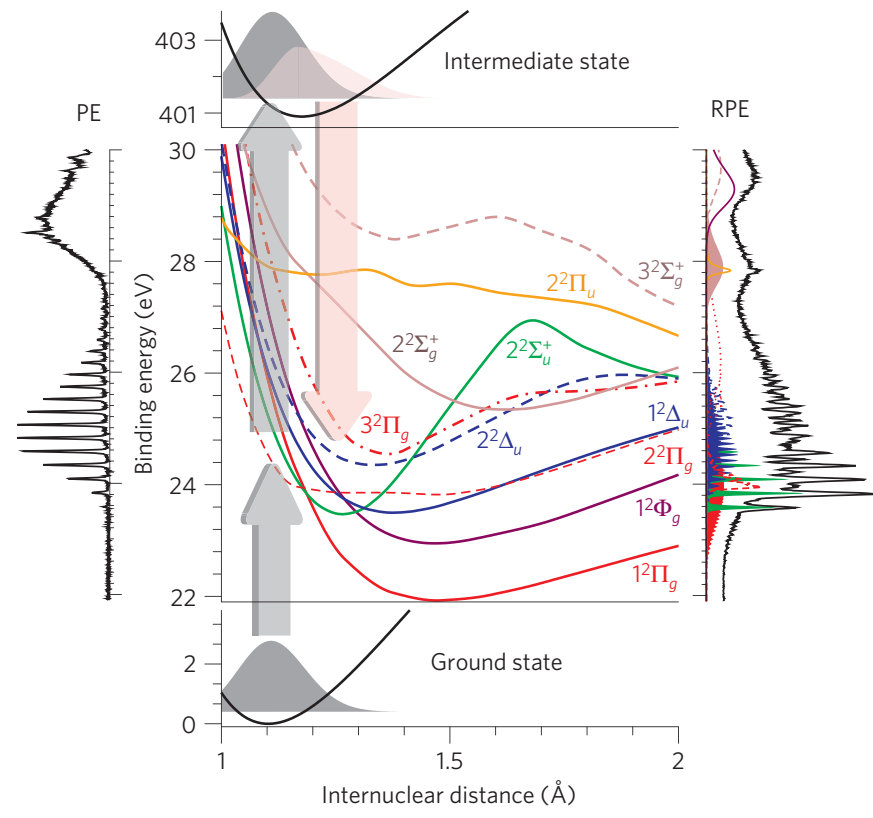

Figure $1 \mid \boldsymbol{A} b$ initio potential-energy curves, direct photoemission (PE) and RPE spectra of $\mathbf{N}_{2}$. Ab initio calculated potential-energy curves of the ground, intermediate (core-excited) and final ionic $\left(\mathrm{N}_{2}{ }^{+}\right)$states are shown in the central panel. A strong congestion of states can be observed around the vertical transition from the zero-point vibrational level of the ground electronic state shown by the ascending grey arrow. In addition to the resulting spectral band overlap, some states are 'dark' in the photoemission spectrum recorded at $100 \mathrm{eV}$ photon energy (left-hand panel). The ground-state nuclear wave packet excited to the intermediate-state potential (grey) continuously undergoes Auger decay (pink) to final ionic states, as indicated by the descending pink arrow. Owing to the nuclear dynamics in the core-excited state, the decay takes place at larger internuclear distances, providing the opportunity to visualize the potentials in a region free of any overlap. Experimental and calculated partial contributions to the RPE spectrum of the nitrogen molecule for the X-ray energy tuned to the lowest vibrational substate of the $1 s^{-1} \pi^{*}$ core-excited state are shown in the right-hand panel. The assignment of the spectra is based on the present $a b$ initio calculations and partially on available photoemission data $23,26,27$

(see Fig. 3b). Indeed, tuning the X-ray energy to higher vibrational substates enables this spectral feature, hidden in the region of overlap, to be 'pulled out' into the lower- $E_{\mathrm{b}}$ energy region, where it becomes available for investigation. This is experimentally very demanding because the RPE intensity drops dramatically for these low-cross-section highly excited states. The photoexcitation crosssection to the $v=6$ vibrational sublevel is so low that it can barely be observed, even in the absorption spectrum shown in Fig. $3 \mathrm{~b}$.

In general, the lifetime of the intermediate state is sufficiently long $\left(5.7 \pm 0.2 \mathrm{fs}\right.$ in the present case $\left.\mathrm{e}^{5}\right)$, and the Auger decay taking place at the left and right classical turning points of the coreexcited-state potential (Fig. 3a) plays the main role in the formation of the RPE profile. Nevertheless, the $E_{\mathrm{b}}$ range corresponding to Auger transitions from the left classical turning point lies well above $24 \mathrm{eV}$ (Fig. 3c) and does not contribute in the region of interest. Therefore, below $24 \mathrm{eV}$, the RPE spectrum mainly builds up from the Auger transitions in the vicinity of the right classical turning point (Fig. 3), which shifts to larger internuclear distances when the excitation energy is increased.

Detuning the X-ray energy from the nominal energy of the vertical transitions has been shown, in previous RPE studies, to be an efficient characterization tool for nuclear dynamics ${ }^{8-15}$. We demonstrate here that it may also represent the key for

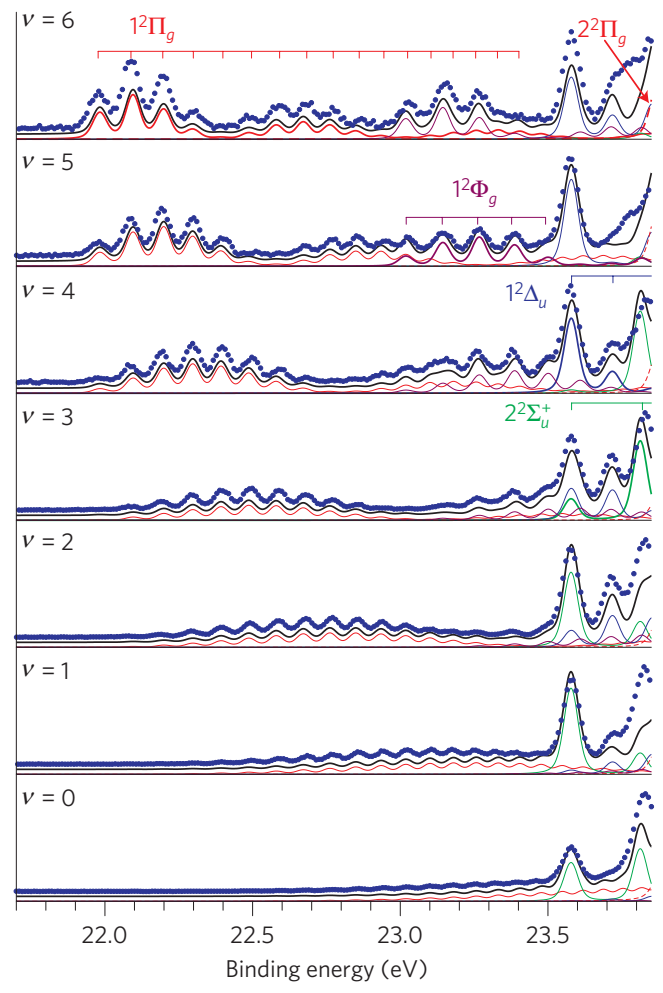

Figure 2 | RPE spectra of nitrogen molecule obtained for a series $v=0-6$ of vibrational substates of the $1 \mathrm{~s}^{-1} \pi^{*}$ core-excited state. The blue dots are the experimental data points, and the calculated RPE spectrum is shown by a black solid line. For the sake of clarity, the experimental and total theoretical RPE spectra are vertically shifted with respect to the partial RPE cross-sections shown by coloured lines. According to our simulations, five ionic states $\left(1^{2} \Pi_{g}, 1^{2} \Phi_{g}, 1^{2} \Delta_{u},{ }^{2} \Sigma_{u}^{+}\right.$and $\left.2^{2} \Pi_{g}\right)$ lie in this spectral region. Previous photoelectron spectroscopy studies ${ }^{23,26-28}$ were only able to detect two of these states $\left({ }^{2} \Sigma_{u}^{+}\right.$and $\left.2^{2} \Pi_{g}\right)$, and earlier RPE measurements of ref. 31 show only a weak unresolved structure tentatively assigned to the $1^{2} \Pi_{g}$ state. Tuning the photon energy in resonance with the higher vibrational sublevels (see Fig. 3b) 'pulls out' the $1^{2} \Pi_{g}$ and $1^{2} \Phi_{g}$ spectral bands from the region of overlap (Fig. 1). Such a refinement of the RPE spectra enables us to reconstruct the potentials of individual electronic states.

molecular-potential reconstruction on the basis of spectroscopic data. Indeed, the increase in excitation energy shifts the right turning point to larger internuclear distances (Fig. 3a) and provides access to the region where final-state potentials are well separated from one another, as can be inferred from Fig. $3 \mathrm{c}$. It is thus possible to avoid the 'contamination' of individual spectral bands from neighbouring, overlapping states. If the $v=0$ substate is selected in the intermediate state, the RPE profile consists of the extensive tail of the $1^{2} \Pi_{g}$ state overlapping with the ${ }^{2} \Sigma_{u}^{+}$and $2^{2} \Pi_{g}$ bands. When increasing the X-ray energy to select higher vibrational substates $(v=1-2)$, the $1^{2} \Pi_{g}$ band progressively shifts out of the region of overlap (Fig. 2). From $v \geq 3$, the $1^{2} \Phi_{g}$ state vibrational progression also becomes available. Moreover, it is fundamental to note that both $1^{2} \Pi_{g}$ and $1^{2} \Phi_{g}$ states can be fully explored down to their ground vibrational substates only when the highest vibrational levels $v=5-6$ are selected in the intermediate state. The visible modulation of the intensity distribution within the vibrational progression of the $1^{2} \Pi_{g}$ state fingerprints accurately the nodal structure of the vibrational wavefunction of the core-excited state, in agreement with the reflection principle-the basis for the mapping technique ${ }^{16}$. The temptation to use the mapping technique to obtain information about molecular potentials has motivated 


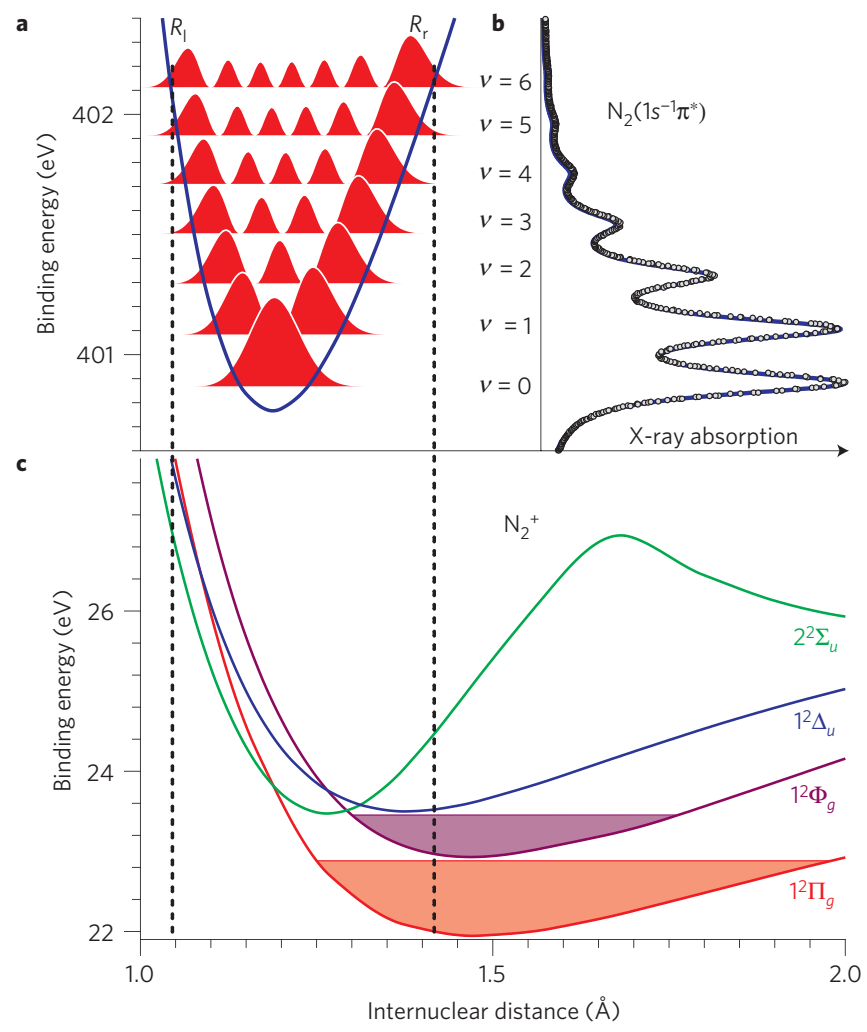

Figure 3 | Controlling the extension of the vibrational wavefunctions in the intermediate core-excited state through the X-ray photon energy. a, Calculated potential of the $1 s^{-1} \pi^{*}$ core-excited state and the squares of the vibrational wavefunctions of the first vibrational substates. Left $\left(R_{\mid}\right)$and right $\left(R_{\mathrm{r}}\right)$ classical turning points are shown for $v=6$. $\mathbf{b}$, Experimental (circles) and theoretical (solid line) N $1 s \rightarrow \pi^{*}$ X-ray photoabsorption spectra of nitrogen molecule. $\mathbf{c}$, Selected final ionic-state potentials that can be explored in the region free of mutual overlap are shown by the shaded areas.

several studies (see for instance refs 13,17), but unfortunately the extracted information was rather poor. Instead, we propose here to accurately reconstruct the final-state potentials using the full vibrationally resolved RPE spectrum. The accuracy of this method is high as soon as a large number of vibrational states is populated. We used the sum of all RPE spectra recorded for the series $v=0-6$ of vibrational substates to maximize the extension of the vibrational progressions observable in the resulting RPE spectrum. These progressions have been fitted following standard procedures described in Supplementary Information. The molecular constants were obtained from the fits, and the potential has been reconstructed using the general Morse function ${ }^{18}$. The same procedure has also been applied to reconstruct the $1^{2} \Phi_{g}$-state potential. The reconstructed potentials for the $1^{2} \Pi_{g}$ and $1^{2} \Phi_{g}$ ionic states are shown together with the $a b$ initio calculated potentials in Fig. 4. It is important to note that remarkably good agreement is obtained with the Rydberg-Klein-Rees-based potential ${ }^{19}$ for the $1^{2} \Pi_{g}$ state not only for the vibrational frequency $\omega_{\mathrm{e}}$ and the anharmonicity parameter, $x_{\mathrm{e}} \omega_{\mathrm{e}}$, but also for the dissociation energy (Supplementary Table S1). As our experiment provides the first observation of the $1^{2} \Phi_{g}$ state we are unable to compare the results (Supplementary Table S1) with independent data. Nevertheless, the reconstructed potential of this newly observed state is also in good agreement with the $a b$ initio potential as shown in Fig. 4. We would like to emphasize that the $1^{2} \Delta_{u}$ band, which becomes visible starting from $v \geq 2$ (Fig. 2), is also observed experimentally for the first time.

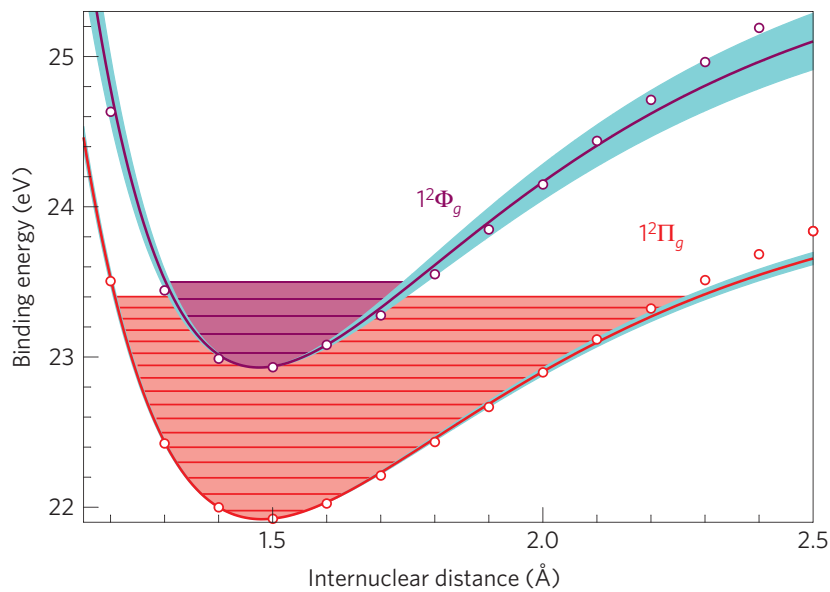

Figure 4 | Comparison between the reconstructed molecular potentials based on ultrahigh-resolution RPE data and $a b$ initio calculated potentials (circles). Solid lines show potential-energy curves of the $1^{2} \Pi_{g}$ and $1^{2} \Phi_{g}$ final ionic states reconstructed from the RPE experimental data using the general Morse function ${ }^{18}: E(r)=E_{\min }+D\left[1+\left(\lambda_{2} e^{-\lambda_{1} r}-\lambda_{1} e^{-\lambda_{2} r}\right) /\left(\lambda_{1}-\lambda_{2}\right)\right]$. Here $r=R-R_{\mathrm{e}}$ and $R_{\mathrm{e}}$ is the bond length at the minimum of the potential as obtained from the fitting of the vibrational progression. The energy interval used for potential reconstruction is delimited by the darker areas. The uncertainty in the reconstructed potential curves (light blue) was obtained by error propagation for the general Morse function using the standard deviations for the spectroscopic constants (see Supplementary Information).

In conclusion, recording ultrahigh-resolution RPE spectra over a wide excitation-energy range offers a unique opportunity to image the potential-energy curves in an extended spatial domain where the X-ray-driven nuclear wave packet in the core-excited state plays the role of a local probe controlled by the photon energy. Tuning the excitation energy is a key feature to overcome the rather common issue of overlapping molecular spectral bands. We show that state-of-the-art RPE spectroscopy can be successfully used to solve the problem of potential reconstruction for excited molecular states directly from experimental data, and to identify such new states, invisible in conventional photoemission spectroscopy. The demonstration was made using a textbook case-the nitrogen molecule. However, we suggest that our method is general and can be extended to larger species and easily transposed to neutral molecular-state potentials by detecting the radiative decay.

\section{Methods}

The experiment was carried out at the PLEIADES beamline ${ }^{6,7}$ at the SOLEIL synchrotron in Saint-Aubin, France. The beamline covers the 7-1,000 eV energy range, with any polarization. Monochromatic light is obtained from a plane grating monochromator with no entrance slit. Typical photon fluxes up to $10^{13}$ photons $^{-1}$ per $0.1 \%$ bandwidth are available and an ultimate resolving power of approximately $10^{5}$ is achievable at $50 \mathrm{eV}$. The measurements were made using a VG-Scienta R4000 electron spectrometer mounted with the electron detection axis vertical. X-ray polarization was set at the magic angle of $54.7^{\circ}$ with respect to this axis. Pure nitrogen (99.998\%) from Air Liquide was used and the pressure in the vacuum chamber was kept constant around $1 \times 10^{-5} \mathrm{mbar}$. The monochromator slit was $40 \mu \mathrm{m}$, corresponding to a photon bandwidth better than $20 \mathrm{meV}$ at $401 \mathrm{eV}$. The electron spectrometer was operated at a $20 \mathrm{eV}$ pass energy, providing a kinetic energy resolution of $15 \mathrm{meV}$. The resulting total instrumental broadening was less than $52 \mathrm{meV}$.

The potential-energy curves of the ground, core-excited and final ionic states of $\mathrm{N}_{2}$ were computed using the configuration-interaction approach implemented in the GSCF3 quantum chemical package ${ }^{20,21}$. We used the basis set and polarization function (10s7pld) of ref. 22 contracted as [5221/52/1]. The full valence configuration interaction for the $2 \sigma_{g}, 3 \sigma_{g}, 2 \sigma_{u}, 3 \sigma_{u}^{*}, 1 \pi_{u}$ and $1 \pi_{g}^{*}$ orbitals, and single and double configuration interaction for external orbitals from the full valence space (namely, second-order configuration interaction), was carried out. The results obtained are in good agreement with previous calculation $\mathrm{s}^{23-25}$ and experiments ${ }^{26-28}$ for $\mathrm{N}_{2}{ }^{+}$. To simulate the RPE spectra, the time-dependent 
wave-packet approach was used ${ }^{29,30}$. The total spectrum has been obtained as a superposition of contributions of the individual electronic states. Calculated spectra were convoluted with appropriate Gaussian functions to account for the experimental broadenings.

Received 22 August 2011; accepted 2 November 2011; published online 4 December 2011; corrected after print 19 November 2015

\section{References}

1. Puschnig, P. et al. Reconstruction of molecular orbital densities from photoemission data. Science 326, 702-706 (2009).

2. Huber, K. P. \& Herzberg, G. Molecular Spectra and Molecular Structure, Vol. 4: Constants of Diatomic Molecules (Van Nostrand Reinhold, 1979).

3. Itatani, J. et al. Tomographic imaging of molecular orbitals. Nature 432, 867-871 (2004).

4. Steele, D. \& Lippincott, E. R. Comparative study of empirical internuclear potential functions. Rev. Mod. Phys. 34, 239-251 (1962).

5. Prince, K. C. et al. A critical comparison of selected $1 \mathrm{~s}$ and $2 \mathrm{p}$ core hole widths. J. Electron Spectrosc. Related Phenom. 101-103, 141-147 (1999).

6. Travnikova, O. et al. Circularly polarized X rays: Another probe of ultrafast molecular decay dynamics. Phys. Rev. Lett. 105, 233001 (2010).

7. Söderström, J. et al. Angle-resolved electron spectroscopy of the resonant Auger decay in xenon with meV energy resolution. New J. Phys. 13, 073014 (2011)

8. Gel'mukhanov, F. \& Ågren, H. Resonant X-ray Raman scattering. Phys. Rep. 312, 87-330 (1999).

9. Piancastelli, M-N. Auger resonant Raman studies of atoms and molecules. J. Electron Spectrosc. Related Phenom. 107, 1-26 (2000).

10. Ueda, K. Core excitation and de-excitation spectroscopies of free atoms and molecules. J. Phys. Soc. Jpn 75, 032001 (2006).

11. Miron, C. \& Morin, P. in Handbook of High-Resolution Spectroscopy (eds Quack, M. \& Merkt, F.) 1655-1690 (John Wiley, 2011).

12. Miron, C. et al. Nuclear motion driven by the Renner-Teller effect as observed in the resonant Auger decay to the X electronic ground state in $\mathrm{N}_{2} \mathrm{O}$. J. Chem. Phys. 115, 864-868 (2001).

13. Miron, C. et al. Mapping potential energy surfaces by core excitation of polyatomic molecules. Chem. Phys. Lett. 359, 48-54 (2002).

14. Miron, C. et al. Vibrational scattering anisotropy generated by multichannel quantum interference. Phys. Rev. Lett. 105, 093002 (2010)

15. Liu, J-C. et al. Multimode resonant Auger scattering from the ethene molecule. J. Phys. Chem. B 115, 5103-5112 (2011).

16. Gel'mukhanov, F. \& Agren, H. X-ray resonant scattering involving dissociative states. Phys. Rev. A 54, 379-393 (1996).

17. Carniato, S. et al. K-L resonant X-ray Raman scattering as a tool for potential energy surface mapping. Chem. Phys. Lett. 439, 402-406 (2007).

18. Lim, T-C. Modification of Morse potential in conventional force fields for applying FPDP parameters. J. Math. Chem. 47, 984-989 (2010).

19. Namioka, T., Yoshino, K. \& Tanaka, Y. Isotope bands and the vibration assignment of the $D^{2} \Pi_{g}-A^{2} \Pi_{u}$ system of $\mathrm{N}_{2}^{+}$. J. Chem. Phys. 39, 2629-2633 (1963).
20. Kosugi, N. \& Kuroda, H. Efficient methods for solving the open-shell SCF problem and for obtaining an initial guess. The one-Hamiltonian and the partial SCF methods. Chem. Phys. Lett. 74, 490-493 (1980).

21. Kosugi, N. Strategies to vectorize conventional SCF-Cl algorithms. Theor. Chim. Acta 72, 149-173 (1987).

22. Huzinaga, S. et al. Physical Sciences Data Vol. 16 (Elsevier, 1984)

23. Baltzer, P., Larsson, M., Karlsson, L., Wannberg, B. \& Göthe, M-C. Inner-valence states of $\mathrm{N}_{2}{ }^{+}$studied by UV photoelectron spectroscopy and configuration-interaction calculations. Phys. Rev. A 46, 5545-5553 (1992).

24. Langhoff, S. R. \& Bauschlicher, C. W. Jr Theoretical study of the first and second negative systems of $\mathrm{N}_{2}^{+}$. J. Chem. Phys. 88, 329-336 (1988).

25. Honjou, N. \& Miyoshi, E. An ab initio study on the electronic structure of the $3^{2} \Sigma_{u}^{+}, 3^{2} \Sigma_{g}^{+}$and $4^{2} \Sigma_{g}^{+}$states of $\mathrm{N}_{2}^{+}$. J. Mol. Struct. 451, 41-49 (1998).

26. Yoshii, H., Tanaka, T., Morioka, Y., Hayaishi, T. \& Hall, R. I. New $\mathrm{N}_{2}{ }^{+}$electronic state in the region of 23-28 eV. J. Mol. Spectrosc. 186, 155-161 (1997).

27. Aoto, T. et al. Inner-valence states of $\mathrm{N}_{2}{ }^{+}$and the dissociation dynamics studied by threshold photoelectron spectroscopy and configuration interaction calculation. J. Chem. Phys. 124, 234306 (2006).

28. Nicolas, C. et al. Dissociative photoionization of $\mathrm{N}_{2}$ in the $24-32 \mathrm{eV}$ photon energy range. J. Phys. B 36, 2239-2251 (2003).

29. Sałek, P., Gel'mukhanov, F. \& Ågren, H. Wave-packet dynamics of resonant $\mathrm{x}$-ray Raman scattering: Excitation near the $\mathrm{Cl}_{\mathrm{II}, \mathrm{III}}$ edge of $\mathrm{HCl}$. Phys. Rev. A 59, 1147-1159 (1999).

30. Sałek, P. A wave-packet technique to simulate resonant X-ray scattering cross sections. Comput. Phys. Commun. 150, 85-98 (2003).

31. Piancastelli, M-N. et al. Electron decay following the $\mathrm{N} 1 s \rightarrow \pi^{*}$ excitation in $\mathrm{N}_{2}$ studied under resonant Raman conditions. J. Electron Spectrosc. Related Phenom. 98-99, 111-120 (1999).

\section{Acknowledgements}

Experiments were carried out at the PLEIADES beamline at SOLEIL Synchrotron, France (proposal number 99090106). We are grateful to J. B. A. Mitchell for his suggestions, to E. Robert for technical assistance and to the SOLEIL staff for smoothly running the facility. The research leading to these results has received funding from the European Union Seventh Framework Programme (FP7/2007-2013) under grant agreement 252781, from Triangle de la physique under contract 2007-010T, from JSPS and from the Swedish Research Council.

\section{Author contributions}

C.M. suggested and planned the experiment. C.N., O.T. and C.M. collected the data, and V.K. and O.T. carried out the data analysis. Y.S., F.G., N.K. and V.K. carried out the theoretical analysis. C.M., F.G. and V.K. wrote the paper and O.T. contributed to figure production. All authors discussed the results and commented on the manuscript.

\section{Additional information}

The authors declare no competing financial interests. Supplementary information accompanies this paper on www.nature.com/naturephysics. Reprints and permissions information is available online at http://www.nature.com/reprints. Correspondence and requests for materials should be addressed to C.M. 


\section{Corrigendum: Imaging molecular potentials using ultrahigh-resolution}

resonant photoemission

Catalin Miron, Christophe Nicolas, Oksana Travnikova, Paul Morin, Yuping Sun, Faris Gel'mukhanov, Nobuhiro Kosugi and Victor Kimberg

Nature Physics 8, 135-138 (2012); published online 4 December 2011; corrected after print 19 November 2015.

In the version of this Letter originally published the state with $E_{\min }=23 \mathrm{eV}$ was mislabelled throughout and should have been labelled $1^{2} \Phi_{g}$. This has been corrected in the online versions 19 November 2015. 